\title{
In Vitro Antagonistic Effect of Gut Bacteriota Isolated from Indigenous Honey Bees and Essential Oils against Paenibacillus Larvae
}

\author{
Miroslava Kačániová ${ }^{1,2, *(\mathbb{D})}$, Margarita Terentjeva ${ }^{3} \mathbb{D}$, Jana Žiarovská 4 \\ and Przemysław Łukasz Kowalczewski ${ }^{5, *(D)}$
}

1 Department of Fruit Science, Viticulture and Enology, Faculty of Horticulture and Landscape Engineering, Slovak University of Agriculture, Tr. A. Hlinku 2, 94976 Nitra, Slovakia

2 Department of Bioenergetics, Food Analysis and Microbiology, Institute of Food Technology and Nutrition, University of Rzeszow, Cwiklinskiej 1, 35-601 Rzeszow, Poland

3 Institute of Food and Environmental Hygiene, Faculty of Veterinary Medicine, Latvia University of Life Sciences and Technologies, K. Helmanaiela 8, LV-3004 Jelgava, Latvia; margarita.terentjeva@llu.lv

4 Department of Plant Genetics and Breeding, Faculty of Agrobiology and Food Resources, Slovak University of Agriculture, Tr. A. Hlinku 2, 94976 Nitra, Slovakia; jana.ziarovska@uniag.sk

5 Department of Food Technology of Plant Origin, Poznań University of Life Sciences, 31 Wojska Polskiego St., 60-624 Poznań, Poland

* Correspondence: miroslava.kacaniova@gmail.com (M.K.); przemyslaw.kowalczewski@up.poznan.pl (P.Ł.K.); Tel.: +421-641-4715 (M.K.); +48-61-848-7297 (P.Ł.K.)

Received: 27 August 2020; Accepted: 12 September 2020; Published: 14 September 2020

check for updates

\begin{abstract}
The aim of study was to isolate and identify the gut bacteria of Apis mellifera and to evaluate antagonistic effect of the bacteriota against Paenibacillus larvae, which causes American foulbrood (AFB) in honeybees. The dilution plating method was used for the quantification of selected microbial groups from digestive tract of bees, with an emphasis on the bacteriota of the bees' intestines. Bacteria were identified using mass spectrometry (MALDI-TOF-MS Biotyper). Overall, five classes, 27 genera and 66 species of bacteria were identified. Genera Lactobacillus (10 species) and Bacillus (8 species) were the most abundant. Gram-negative bacteria were represented with 16 genera, whereas Gram-positive with 10 genera. Delftia acidovorans and Escherichia coli were the most abundant in the digestive tract of honey bee. Resistance to a selection of antimicrobials was assessed for the bacterial isolates from bee gut and confirmed against all antimicrobials included in the study, with the exception of cefepime. Lactobacillus spp., especially L. kunkeei, L. crispatus and L. acidophilus. showed the strongest antimicrobial activity against $P$. larvae, the causal pathogen of AFB. Antimicrobial activity of essential oils against isolated bacteria and two isolates of P. larvae were assessed. Application of a broad selection of plant essential oils indicated that Thymus vulgaris had the highest antimicrobial activity against P. larvae.
\end{abstract}

Keywords: Lactobacillus spp.; rectum; intestine; antimicrobial activity; antimicrobial resistance; essential oils

\section{Introduction}

The digestive tract of the worker bee is inhabited with a variety of microorganisms diverse in their morphology, physiology and metabolism. The microbiota of digestive tract consists of yeasts (1\%), Gram-positive bacteria (29\%) and Gram-negative and gram-variable bacteria (70\%) [1]. The first research on microbiota of digestive tract of bees had been published in the beginning of the 20th 
century and Lactobacillus rigidus apis, Lactobacillus constellatus and Bacillus influenzoides apis were found the main representatives of digestive tract microbiota. Subsequent reports on microflora studies of bees and microorganisms in their diet were published in the 1960s [2,3]. It has been agreed that the only probiotic bacteria species present belonged to Bifidobacteria [4].

American foulbrood (AFB) is a disease caused by aerobic to microaerophilic, Gram-positive, spore-forming rod, Paenibacillus larvae. The disease causes huge economic losses to beekeepers around the world [5]. P. larvae affects honey bee larvae in period when it takes food, rendering the bee larvae more susceptible between 12 to $48 \mathrm{~h}$ of life. Bacterial spores germinate in the gut of larvae, bacteria multiply and kill the larvae at pre-pupal or pupal stage. Infected larvae are settled at the bottom of the cells with sunken sealed brood appearance. The disease is highly contagious as more than 2.5 billion oval spores could be produced in 10 days. AFB does not affect the adult bees, but they facilitate the spread of infection within a colony [6].

The use of antimicrobials in beekeeping is permitted in the United States and is also used in South America and some East Asian countries. In the European Union, the application of antimicrobials in beekeeping is banned in some countries [7].

In recent years, there has been a growing interest in application of natural substances, including for pathogen and pest control: chemical compounds of plant secondary metabolism, extracts or vegetable oils supporting green consumer behavior and healthy lifestyles trends. The diversity of plants stimulates the search and research of new plant-based chemical compounds. Some of identified compounds share antimicrobial activity against pathogenic microorganisms and have even appeared in controlled clinical trials [8]. In particular, the essential oils and mixtures of mono- and sesquiterpenes are known for their strong antimicrobial activity. The possible applications include, inter alia, food production, medicines or cosmetics industries [9].

Therefore, the aims of this study were: (i) to isolate and identify bacteria from the digestive tract of adult honeybee workers (Apis mellifera), (ii) evaluate the antagonistic effects of selected bacteria from the bee gut against the bacteria $P$. larvae and (iii) detect antimicrobial activity of essential oils against P. larvae.

\section{Results}

\subsection{Bacteriota of Adult Worker Bees (Apis mellifera)}

Groups of bacteria isolated from the digestive tract of summer and winter adult worker bees are shown in Table 1. The highest counts of aerobic microorganisms were found in the intestine of winter bees ( $5.39 \pm 0.14 \log \mathrm{cfu} / \mathrm{g})$ and the lowest in the rectum of summer bees $(4.48 \pm 0.13 \mathrm{log} \mathrm{cfu} / \mathrm{g})$. The total counts of anaerobic microorganisms ranged from $8.12 \pm 0.06$ in the intestine of summer bees to $9.25 \pm 0.15 \mathrm{log} \mathrm{cfu} / \mathrm{g}$ in the rectum of winter bees. Anaerobic Gram-positive microorganisms counts ranged from $6.13 \pm 0.09$ for summer bees in the intestine to $7.10 \pm 0.12 \mathrm{log} \mathrm{cfu} / \mathrm{g}$ for winter bees in the rectum. The lowest counts of Bacillus spp. were found in the intestine of winter bees $(2.48 \pm 0.09 \mathrm{log} \mathrm{cfu} / \mathrm{g})$ and the highest were found in the winter bees in the rectum $(3.53 \pm 0.07 \mathrm{log} \mathrm{cfu} / \mathrm{g})$. The lowest counts of Lactobacillus spp. were found in the intestine of winter bees $(7.14 \pm 0.06)$ whereas the highest were found in the rectum of winter bees $(8.27 \pm 0.11)$. The coliform bacteria counts were the highest in the rectum of the winter bees $(3.57 \pm 0.13)$ whereas the lowest counts were in the intestines of the winter bees $(2.52 \pm 0.11)$. There were statistically significant differences among all groups of microorganisms ( $p \leq 0.05, p \leq 0.01)$. 
Table 1. Isolated bacteriota of adult worker honeybee guts in in $\log \mathrm{cfu} / \mathrm{g}$ (mean $\pm \mathrm{SD})$.

\begin{tabular}{ccccc}
\hline & \multicolumn{2}{c}{ Bee Gut from Intestine } & \multicolumn{2}{c}{ Bee Gut from Rectum } \\
\hline & Winter Bees Samples & Summer Bees Samples & Winter Bees Samples & Summer Bees Samples \\
\hline TCAM $^{*}$ & $5.39 \pm 0.14^{\mathrm{a}}$ & $5.03 \pm 0.16^{\mathrm{ab}}$ & $5.00 \pm 0.22^{\mathrm{abc}}$ & $4.48 \pm 0.13^{\mathrm{abc}}$ \\
TCANM $^{\mathrm{a}}$ & $8.38 \pm 0.11^{\mathrm{a}}$ & $8.12 \pm 0.06^{\mathrm{b}}$ & $9.25 \pm 0.15^{\mathrm{ab}}$ & $9.05 \pm 0.09^{\mathrm{ab}}$ \\
AG $^{+}$ & $6.49 \pm 0.13^{\mathrm{a}}$ & $6.13 \pm 0.09^{\mathrm{ab}}$ & $7.10 \pm 0.12^{\mathrm{abc}}$ & $6.77 \pm 0.11^{\mathrm{abc}}$ \\
BS & $2.48 \pm 0.09^{\mathrm{a}}$ & $3.43 \pm 0.16^{\mathrm{ab}}$ & $3.53 \pm 0.07^{\mathrm{ac}}$ & $3.22 \pm 0.10^{\mathrm{abc}}$ \\
LS & $7.14 \pm 0.06^{\mathrm{a}}$ & $7.66 \pm 0.14^{\mathrm{ab}}$ & $8.27 \pm 0.11^{\mathrm{ab}}$ & $8.12 \pm 0.06^{\mathrm{ab}}$ \\
PS & $2.55 \pm 0.06^{\mathrm{a}}$ & $2.29 \pm 0.13^{\mathrm{ab}}$ & $3.12 \pm 0.07^{\mathrm{abc}}$ & $2.85 \pm 0.15^{\mathrm{abc}}$ \\
ES & $3.21 \pm 0.08^{\mathrm{a}}$ & $3.42 \pm 0.12^{\mathrm{ab}}$ & $2.24 \pm 0.10^{\mathrm{abc}}$ & $2.53 \pm 0.15^{\mathrm{abc}}$ \\
SS & $3.22 \pm 0.09^{\mathrm{a}}$ & $3.45 \pm 0.08^{\mathrm{ab}}$ & $2.56 \pm 0.19^{\mathrm{abc}}$ & $2.25 \pm 0.07^{\mathrm{abc}}$ \\
CB & $2.52 \pm 0.11^{\mathrm{a}}$ & $3.25 \pm 0.13^{\mathrm{ab}}$ & $3.57 \pm 0.13^{\mathrm{abc}}$ & $3.37 \pm 0.14^{\mathrm{ac}}$ \\
\hline
\end{tabular}

* TCAM-total counts of aerobic microorganisms, TCANM-total counts of anaerobic microorganisms, $\mathrm{AG}^{+}$-anaerobic Gram-positive bacteria, BS-Bacillus spp., LS-Lactobacillus spp., PS-Pseudomonas spp., ES-Enterococcus spp., SS-Staphylococcus spp., CB-coliform bacteria. ${ }^{\mathrm{a}, \mathrm{b}, \mathrm{c}}$ same letters in the raw show statistically significant differences among the groups.

\subsection{Isolated Bacteria from Bees Gut}

A total of five classes of bacteria were obtained from the gut of the honey bee: Actinobacteria, Alphaproteobacteria, Betaproteobacteria, Firmicutes and Gammaproteobacteria. A total of 27 genera were isolated from the honey bee bacteriota: Aeromonas, Arthrobacter, Bacillus, Citrobacter, Delftia, Enterobacter, Enterococcus, Escherichia, Fructobacillus, Hafnia, Klebsiella, Kocuria, Lactobacillus, Lactococcus, Microbacterium, Moraxella, Morganella, Paenibacillus, Pantotea, Proteus, Pseudomonas, Rahnella, Ralstonia, Raoultella, Serratia, Sphingomonas and Staphylococcus. A total of 66 species were isolated from bees, of which the genus Lactobacillus represented by 10 species and the genus Bacillus by eight species were the most numerous (Table 2).

Table 2. Isolated species of adult worker honeybee bacteriota from gastrointestinal tract.

\begin{tabular}{ccc}
\hline Class & Genus & Species \\
\hline Gammaproteobacteria & Aeromonas & Aeromonas salmonicida \\
Actinobacteria & Arthrobacter & Arthrobacter tumbae \\
Firmicutes & Bacillus & Bacillus cereus \\
Firmicutes & Bacillus & Bacillus circulans \\
Firmicutes & Bacillus & Bacillus licheniformis \\
Firmicutes & Bacillus & Bacillus megaterium \\
Firmicutes & Bacillus & Bacillus oleronius \\
Firmicutes & Bacillus & Bacillus spp. \\
Firmicutes & Bacillus & Bacillus subtilis \\
Firmicutes & Bacillus & Bacillus thuringiensis \\
Gammaproteobacteria & Citrobacter & Citrobacter spp. \\
Gammaproteobacteria & Citrobacter & Citrobacter braakii \\
Gammaproteobacteria & Citrobacter & Citrobacter koseri \\
Betaproteobacteria & Delftia & Delftia acidovorans \\
Gammaproteobacteria & Enterobacter & Enterobacter aerogenes \\
Gammaproteobacteria & Enterobacter & Enterobacter clocae \\
Gammaproteobacteria & Enterobacter & Enterobacter kobei \\
Firmicutes & Enterococcus & Enterococcus cloacae \\
Firmicutes & Enterococcus & Enterococcus faecalis \\
Gammaproteobacteria & Escherichia & Escherichia coli \\
Firmicutes & Fructobacillus & Fructobacillus fructosus \\
Gammaproteobacteria & Hafnia & Hafnia alvei \\
Gammaproteobacteria & Klebsiella & Klebsiella aerogenes \\
Gammaproteobacteria & Klebsiella & Klebsiella oxytoca \\
Gammaproteobacteria & Klebsiella & Klebsiella pneumoniae \\
Gammaproteobacteria & Klebsiella & Klebsiella variicola \\
\hline
\end{tabular}


Table 2. Cont.

\begin{tabular}{|c|c|c|}
\hline Class & Genus & Species \\
\hline Actinobacteria & Kocuria & Kocuria kristinae \\
\hline Firmicutes & Lactobacillus & Lactobacillus acidophilus \\
\hline Firmicutes & Lactobacillus & Lactobacillus agilis \\
\hline Firmicutes & Lactobacillus & Lactobacillus apis \\
\hline Firmicutes & Lactobacillus & Lactobacillus brevis \\
\hline Firmicutes & Lactobacillus & Lactobacillus crispatus \\
\hline Firmicutes & Lactobacillus & Lactobacillus jensenii \\
\hline Firmicutes & Lactobacillus & Lactobacillus kunkeei \\
\hline Firmicutes & Lactobacillus & Lactobacillus mellis \\
\hline Firmicutes & Lactobacillus & Lactobacillus plantarum \\
\hline Firmicutes & Lactobacillus & Lactobacillus spp. \\
\hline Firmicutes & Lactococcus & Lactococcus garvieae \\
\hline Firmicutes & Lactococcus & Lactococcus lactis \\
\hline Actinobacteria & Microbacterium & Microbacterium pumilum \\
\hline Actinobacteria & Microbacterium & Microbacterium testaceum \\
\hline Gammaproteobacteria & Moraxella & Moraxella spp. \\
\hline Gammaproteobacteria & Morganella & Morganella morgani \\
\hline Firmicutes & Paenibacillus & Paenibacillus larvae \\
\hline Gammaproteobacteria & Pantotea & Pantotea agglomerans \\
\hline Gammaproteobacteria & Pantotea & Pantotea ananatis \\
\hline Gammaproteobacteria & Pantotea & Pantotea vagans \\
\hline Gammaproteobacteria & Proteus & Proteus mirabilis \\
\hline Gammaproteobacteria & Pseudomonas & Pseudomonas marginalis \\
\hline Gammaproteobacteria & Pseudomonas & Pseudomonas oryzihabitans \\
\hline Gammaproteobacteria & Pseudomonas & Pseudomonas putida \\
\hline Gammaproteobacteria & Rahnella & Rahnella aquatilis \\
\hline Gammaproteobacteria & Rahnella & Rahnella terrigena \\
\hline Betaproteobacteria & Ralstonia & Ralstonia picketii \\
\hline Gammaproteobacteria & Raoultella & Raoultella ornithinolytica \\
\hline Gammaproteobacteria & Raoultella & Raoultella planticola \\
\hline Gammaproteobacteria & Serratia & Serratia fonticola \\
\hline Gammaproteobacteria & Serratia & Serratia liquefaciens \\
\hline Gammaproteobacteria & Serratia & Serratia marcescens \\
\hline Alphaproteobacteria & Sphingomonas & Sphingomonas parapaucimobilis \\
\hline Alphaproteobacteria & Sphingomonas & Sphingomonas melonis \\
\hline Firmicutes & Staphylococcus & Staphylococcus capitis \\
\hline Firmicutes & Staphylococcus & Staphylococcus epidermidis \\
\hline Firmicutes & Staphylococcus & Staphylococcus hemolyticus \\
\hline Firmicutes & Staphylococcus & Staphylococcus hominis \\
\hline Firmicutes & Staphylococcus & Staphylococcus warneri \\
\hline
\end{tabular}

In total, there were 10 genera of the Gram-positive and 16 genera of the Gram-negative bacteria isolated in the study. MALDI-TOF-MS Biotyper identification score for Lactococcus garvieae ranged from 2.015 to 2.026, Kocuria kristinae from 2.035 to 2.563, Staphylococcus capitis from 2.035 to 2.503, Staphylococcus epidermidis from 2.050 to 2.445, Staphylococcus hemolyticus from 2.041 to 2.341, Staphylococcus hominis from 2.150 to 2.345, Staphylococcus warneri from 2.053 to 2.545, Hafnia alvei from 2.296 to 2.563, Morganella morganii from 2.198 to 2.578, Pantoea ananatis from 2.196 to 2.363, Pantoea agglomerans ranged 2.371 to 2.466 , Raoultella ornithinolytica from 2.051 to 2.550, Raoultella planticola from 2.198 to 2.428 and Serratia fonticola from 2.190 to 2.251, indicating reliable identification of bacterial species. Similarly, high scores were achieved for the other identified species. From the taxonomic point of view, $42.8 \%$ of isolates belonged to the class Gammaproteobacteria, whereas $43.9 \%$ to Firmicutes, $4.8 \%$ to Betaproteobacteria, $4.3 \%$ to Actinobacteria and $4.2 \%$ to the class Alphaproteobacteria (Figures 1-3). Isolates of Gram-negative bacteria belonged to the families Aeromonadaceae, Comamonadaceae, Enterobacteriaceae, Pseudomonadaceae, Ralstoniaceae and 
Sphingomonadaceae of Proteobacteria phylum. Gram-positive bacteria belonged to the families of Bacillaceae, Enterococcaceae, Lactobacillaceae, Lactococcaceae, Microbacteriaceae, Micrococcaceae, Paenibacillaceae, Staphylococcaceae of phyla Actinobacteria and Firmicutes.

A total of 66 species of bacteria from the digestive tract of bees were isolated, of which 33 were Gram-positive and 33 Gram-negative. Escherichia coli was isolated most frequently from all samples tested, but P. larvae was isolated from only one sample (Table 3).

Table 3. Frequency of isolated bacteriota (\%) detected in the samples of bee digestive tract.

\begin{tabular}{|c|c|c|}
\hline Species & No. of Isolates/No. of Samples & No. of Positive Samples (\%) \\
\hline Aeromonas salmonicida & $15 / 12$ & 6.00 \\
\hline Arthrobacter tumbae & $21 / 15$ & 7.50 \\
\hline Bacillus cereus & $51 / 25$ & 12.50 \\
\hline Bacillus circulans & $35 / 10$ & 5.00 \\
\hline Bacillus licheniformis & $64 / 25$ & 12.50 \\
\hline Bacillus megaterium & $128 / 96$ & 48.00 \\
\hline Bacillus oleronius & $25 / 20$ & 10.00 \\
\hline Bacillus spp. & $125 / 52$ & 26.00 \\
\hline Bacillus subtilis & $56 / 35$ & 17.50 \\
\hline Bacillus thuringiensis & $68 / 42$ & 21.00 \\
\hline Citrobacter spp. & $188 / 112$ & 56.00 \\
\hline Citrobacter braakii & $37 / 15$ & 7.50 \\
\hline Citrobacter koseri & $60 / 30$ & 15.00 \\
\hline Delftia acidovorans & $150 / 200$ & 100.00 \\
\hline Enterobacter aerogenes & $136 / 110$ & 55.00 \\
\hline Enterobacter clocae & $126 / 99$ & 49.50 \\
\hline Enterobacter kobei & $59 / 32$ & 16.00 \\
\hline Enterococcus cloacae & $56 / 15$ & 7.50 \\
\hline Enterococcus faecalis & $150 / 100$ & 50.00 \\
\hline Escherichia coli & $350 / 200$ & 100.00 \\
\hline Fructobacillus fructosus & $29 / 11$ & 5.50 \\
\hline Hafnia alvei & $218 / 169$ & 84.50 \\
\hline Klebsiella aerogenes & $59 / 28$ & 14.00 \\
\hline Klebsiella oxytoca & $98 / 58$ & 29.00 \\
\hline Klebsiella pneumoniae & $36 / 12$ & 6.00 \\
\hline Klebsiella variicola & $45 / 15$ & 7.50 \\
\hline Kocuria kristinae & $186 / 125$ & 62.50 \\
\hline Lactobacillus acidophilus & $64 / 30$ & 15.00 \\
\hline Lactobacillus agilis & $55 / 20$ & 10.00 \\
\hline Lactobacillus apis & $123 / 69$ & 34.50 \\
\hline Lactobacillus brevis & $150 / / 100$ & 50.00 \\
\hline Lactobacillus crispatus & $164 / / 88$ & 44.00 \\
\hline Lactobacillus jensenii & $15 / 10$ & 5.00 \\
\hline Lactobacillus kunkeei & $135 / 120$ & 60.00 \\
\hline Lactobacillus mellis & $64 / 35$ & 17.50 \\
\hline Lactobacillus plantarum & $95 / 80$ & 40.00 \\
\hline Lactobacillus spp. & $167 / 150$ & 75.00 \\
\hline Lactococcus garvieae & $121 / 90$ & 45.00 \\
\hline Lactococcus lactis & $68 / 39$ & 19.50 \\
\hline Microbacterium pumilum & $15 / 5$ & 2.50 \\
\hline Microbacterium testaceum & $25 / 10$ & 5.00 \\
\hline Moraxella spp. & $55 / 15$ & 7.50 \\
\hline Morganella morgani & $115 / 100$ & 50.00 \\
\hline Paenibacillus larvae & $1 / 1$ & 0.50 \\
\hline Pantotea agglomerans & $52 / 40$ & 20.00 \\
\hline Pantotea ananatis & $65 / 30$ & 15.00 \\
\hline Pantotea vagans & $87 / 58$ & 29.00 \\
\hline Proteus mirabilis & $120 / 95$ & 47.50 \\
\hline Pseudomonas marginalis & $12 / 3$ & 1.50 \\
\hline
\end{tabular}


Table 3. Cont.

\begin{tabular}{ccc}
\hline Species & No. of Isolates/No. of Samples & No. of Positive Samples (\%) \\
\hline Pseudomonas oryzihabitans & $65 / 50$ & 25.00 \\
Pseudomonas putida & $35 / 15$ & 7.50 \\
Rahnella aquatilis & $65 / 40$ & 20.00 \\
Rahnella terrigena & $35 / 22$ & 11.00 \\
Ralstonia picketii & $126 / 110$ & 55.00 \\
Raoultella ornithinolytica & $69 / 52$ & 26.00 \\
Raoultella planticola & $35 / 15$ & 7.50 \\
Serratia fonticola & $95 / 95$ & 47.50 \\
Serratia liquefaciens & $87 / 58$ & 29.00 \\
Serratia marcescens & $64 / 30$ & 15.00 \\
Sphingomonas parapaucimobilis & $125 / 100$ & 50.00 \\
Sphingomonas melonis & $120 / 60$ & 30.00 \\
Staphylococcus capitis & $136 / 120$ & 60.00 \\
Staphylococcus epidermidis & $168 / 62$ & 31.00 \\
Staphylococcus haemolyticus & $58 / 35$ & 17.50 \\
Staphylococcus hominis & $112 / 90$ & 45.00 \\
Staphylococcus warneri & $64 / 52$ & 26.00 \\
\hline
\end{tabular}

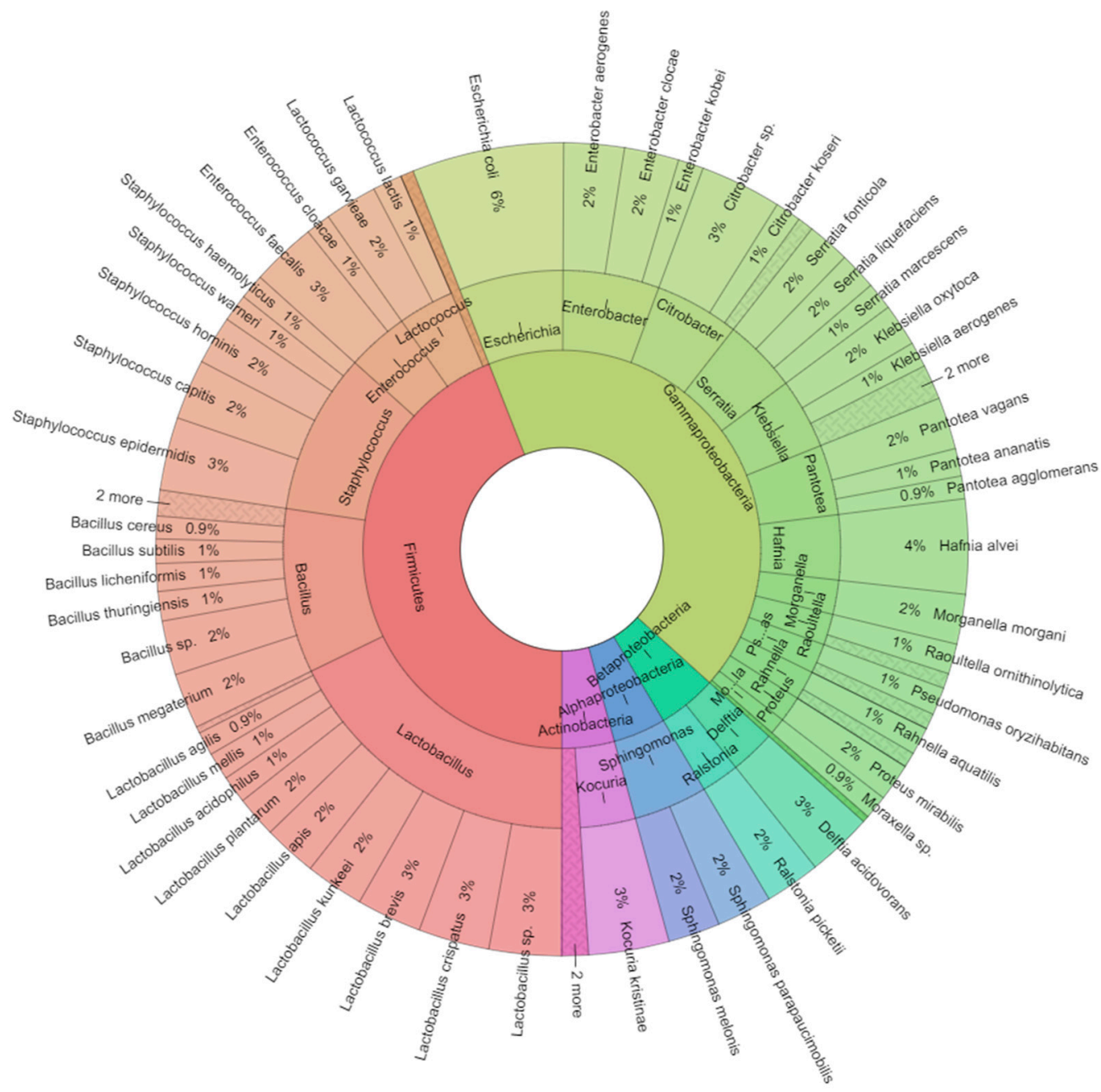

Figure 1. Krona RSF display of total bacteriota isolated from bee digestive tracts. Presented are the frequencies of detected species, genera and classes, from the outer ring inwards. 


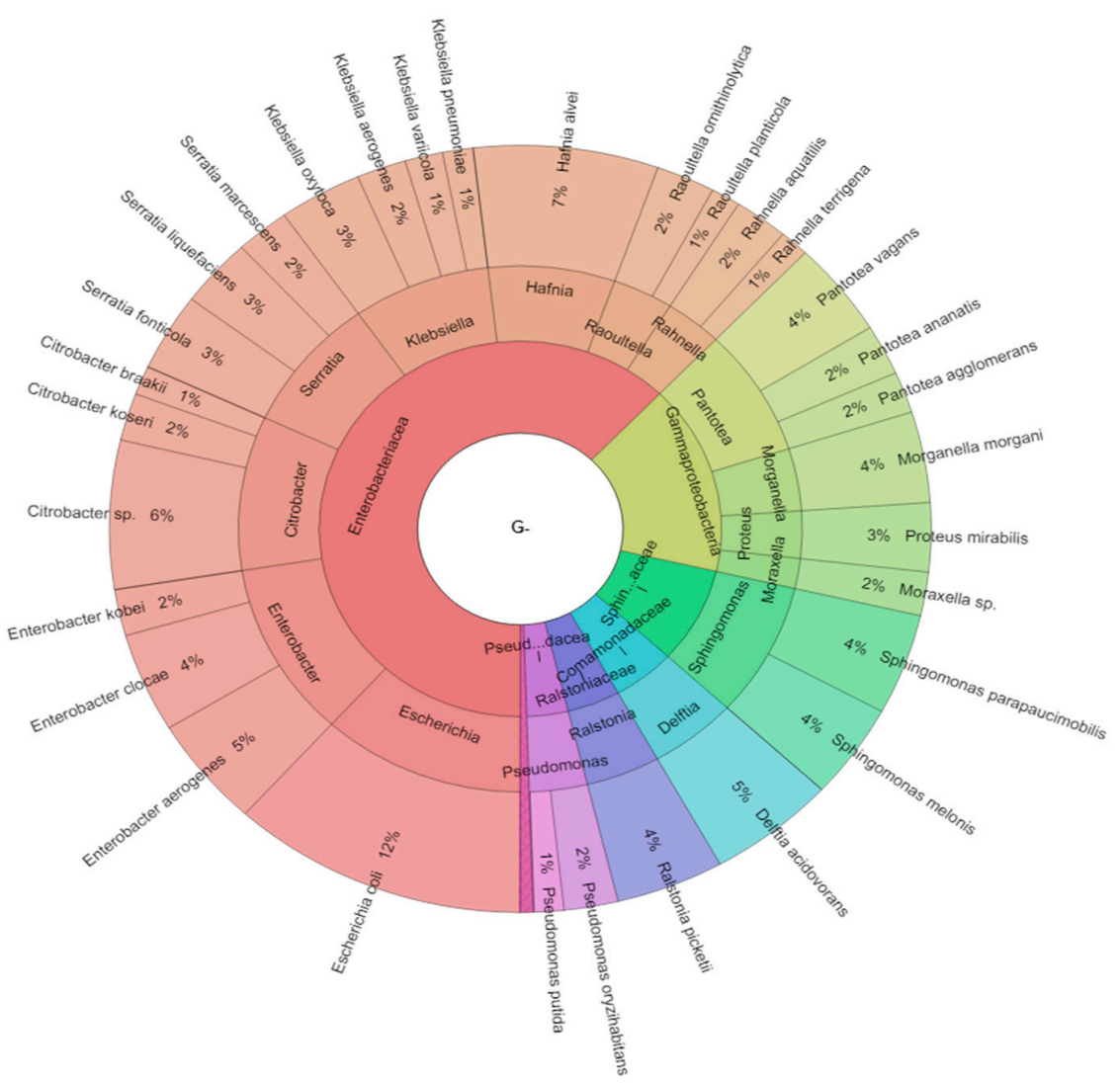

Figure 2. Krona RSF display of Gram-negative bacteriota isolated from bee digestive tracts. Presented are the frequencies of detected species, genera and classes, from the outer ring inwards.

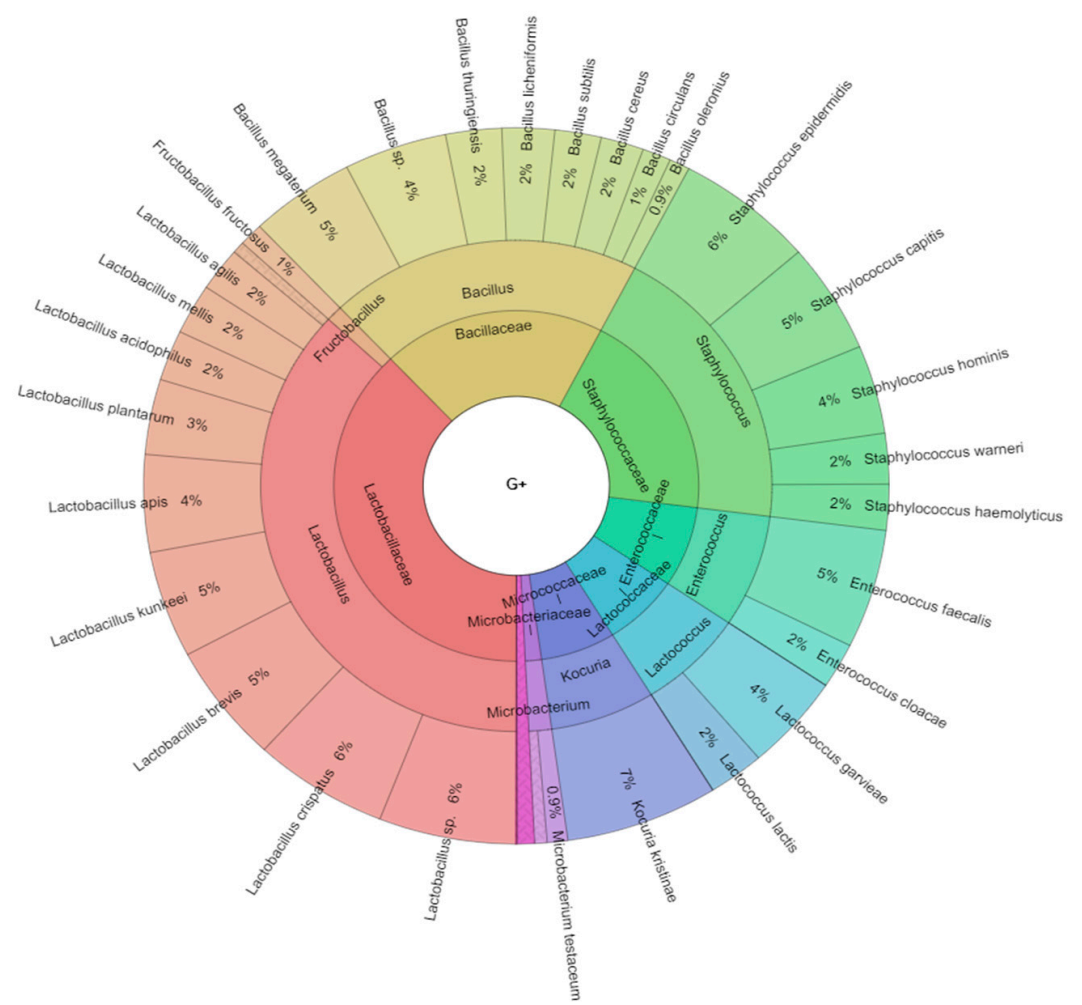

Figure 3. Krona RSF display of Gram-positive bacteriota isolated from bee digestive tracts. Presented are the frequencies of detected species, genera and classes, from the outer ring inwards. 


\subsection{Antibiotic Resistance of A. mellifera Gut Bacteriota}

A total of 5789 isolates were isolated from the digestive tract of 200 bees. Gram-positive and Gram-negative bacteria showed antimicrobial resistance to various classes of antimicrobials (Table 4).

Table 4. Antimicrobial resistance of bacteria isolated from bee digestive tracts.

\begin{tabular}{|c|c|c|c|c|}
\hline Antimicrobial & CEF & CIP & - & - \\
\hline Resistance/Sensitivity & $\mathrm{R} / \mathrm{S}$ & $\mathrm{R} / \mathrm{S}$ & - & - \\
\hline Aeromonas salmonicida & $0 / 15$ & $0 / 15$ & - & - \\
\hline Arthrobacter tumbae & ND & ND & - & - \\
\hline Bacillus cereus & ND & ND & - & - \\
\hline Bacillus circulans & ND & ND & - & - \\
\hline Bacillus licheniformis & ND & ND & - & - \\
\hline Bacillus megaterium & ND & ND & - & - \\
\hline Bacillus oleronius & ND & ND & - & - \\
\hline Bacillus spp. & ND & ND & - & - \\
\hline Bacillus subtilis & ND & ND & - & - \\
\hline \multirow[t]{3}{*}{ Bacillus thuringiensis } & ND & ND & - & - \\
\hline & TIC & IMI & $\mathrm{CIP}$ & $\mathrm{CHL}$ \\
\hline & $\mathrm{R} / \mathrm{S}$ & $\mathrm{R} / \mathrm{S}$ & $\mathrm{R} / \mathrm{S}$ & $\mathrm{R} / \mathrm{S}$ \\
\hline Citrobacter spp. & $8 / 188$ & $25 / 188$ & $0 / 188$ & $45 / 188$ \\
\hline Citrobacter braakii & $6 / 37$ & $15 / 37$ & $5 / 37$ & $10 / 37$ \\
\hline Citrobacter koseri & $16 / 60$ & $10 / 60$ & $5 / 60$ & $14 / 60$ \\
\hline \multirow[t]{3}{*}{ Delftia acidovorans } & ND & ND & ND & ND \\
\hline & $\mathrm{TIC}$ & IMI & CIP & CHL \\
\hline & $\mathrm{R} / \mathrm{S}$ & $\mathrm{R} / \mathrm{S}$ & $\mathrm{R} / \mathrm{S}$ & $\mathrm{R} / \mathrm{S}$ \\
\hline Enterobacter aerogenes & $61 / 136$ & $25 / 136$ & $10 / 136$ & $22 / 136$ \\
\hline Enterobacter clocae & $28 / 126$ & $5 / 136$ & $1 / 136$ & $6 / 136$ \\
\hline \multirow[t]{3}{*}{ Enterobacter kobei } & 9/59 & $5 / 59$ & $0 / 59$ & $0 / 59$ \\
\hline & IMI & TEI & TIG & - \\
\hline & $\mathrm{R} / \mathrm{S}$ & $\mathrm{R} / \mathrm{S}$ & $\mathrm{R} / \mathrm{S}$ & - \\
\hline Enterococcus cloacae & $5 / 56$ & $6 / 56$ & $11 / 56$ & - \\
\hline \multirow[t]{3}{*}{ Enterococcus faecalis } & $58 / 150$ & $10 / 150$ & $25 / 150$ & - \\
\hline & TIC & IMI & CIP & CHL \\
\hline & $\mathrm{R} / \mathrm{S}$ & $\mathrm{R} / \mathrm{S}$ & $\mathrm{R} / \mathrm{S}$ & $\mathrm{R} / \mathrm{S}$ \\
\hline Escherichia coli & $53 / 350$ & $26 / 350$ & $12 / 350$ & $10 / 350$ \\
\hline Fructobacillus fructosus & ND & ND & ND & ND \\
\hline Hafnia alvei & $15 / 218$ & $12 / 218$ & $5 / 218$ & $5 / 218$ \\
\hline Klebsiella aerogenes & $42 / 59$ & $25 / 59$ & $15 / 59$ & $5 / 59$ \\
\hline Klebsiella oxytoca & $63 / 98$ & $35 / 98$ & $15 / 98$ & $10 / 98$ \\
\hline Klebsiella pneumoniae & $14 / 36$ & $10 / 36$ & $5 / 36$ & $1 / 36$ \\
\hline Klebsiella variicola & $5 / 45$ & $10 / 45$ & $4 / 45$ & $5 / 45$ \\
\hline \multirow[t]{3}{*}{ Kocuria kristinae } & ND & ND & ND & ND \\
\hline & AMP & IMI & MER & CHL \\
\hline & $\mathrm{R} / \mathrm{S}$ & $\mathrm{R} / \mathrm{S}$ & $\mathrm{R} / \mathrm{S}$ & $\mathrm{R} / \mathrm{S}$ \\
\hline Lactobacillus acidophilus & $4 / 64$ & $0 / 64$ & $0 / 64$ & $0 / 64$ \\
\hline Lactobacillus agilis & $2 / 55$ & $3 / 55$ & $2 / 55$ & $0 / 55$ \\
\hline Lactobacillus apis & $16 / 123$ & $10 / 123$ & $8 / 123$ & $5 / 123$ \\
\hline Lactobacillus brevis & $15 / 150$ & $20 / 150$ & $10 / 150$ & $15 / 150$ \\
\hline Lactobacillus crispatus & $25 / 164$ & $38 / 164$ & $5 / 164$ & $6 / 164$ \\
\hline Lactobacillus jensenii & $0 / 15$ & $0 / 15$ & $0 / 15$ & $0 / 15$ \\
\hline Lactobacillus kunkeei & $52 / 135$ & $25 / 135$ & $15 / 135$ & $10 / 135$ \\
\hline Lactobacillus mellis & $2 / 64$ & $0 / 64$ & $1 / 64$ & $0 / 64$ \\
\hline Lactobacillus plantarum & $50 / 95$ & 20/95 & $10 / 95$ & $10 / 95$ \\
\hline
\end{tabular}


Table 4. Cont.

\begin{tabular}{|c|c|c|c|c|}
\hline Antimicrobial & CEF & CIP & - & - \\
\hline Lactobacillus spp. & $0 / 167$ & $0 / 167$ & $0 / 167$ & $0 / 167$ \\
\hline Lactococcus garvieae & ND & ND & ND & ND \\
\hline Lactococcus lactis & ND & ND & ND & ND \\
\hline Microbacterium pumilum & ND & ND & ND & ND \\
\hline Microbacterium testaceum & ND & ND & ND & ND \\
\hline \multirow[t]{3}{*}{ Moraxella spp. } & ND & ND & ND & ND \\
\hline & TIC & IMI & CIP & CHL \\
\hline & $\mathrm{R} / \mathrm{S}$ & $\mathrm{R} / \mathrm{S}$ & $\mathrm{R} / \mathrm{S}$ & $\mathrm{R} / \mathrm{S}$ \\
\hline Morganella morgani & $65 / 115$ & $35 / 115$ & $25 / 115$ & $15 / 115$ \\
\hline Paenibacillus larvae & ND & ND & ND & ND \\
\hline Pantotea agglomerans & $15 / 52$ & $15 / 52$ & $10 / 52$ & $10 / 52$ \\
\hline Pantotea ananatis & $10 / 65$ & $15 / 65$ & $15 / 65$ & $10 / 65$ \\
\hline Pantotea vagans & $37 / 87$ & $30 / 87$ & $15 / 87$ & $10 / 87$ \\
\hline \multirow[t]{3}{*}{ Proteus mirabilis } & $25 / 120$ & $15 / 120$ & $16 / 120$ & $10 / 120$ \\
\hline & TIC & IMI & CIP & TOB \\
\hline & $\mathrm{R} / \mathrm{S}$ & $\mathrm{R} / \mathrm{S}$ & $\mathrm{R} / \mathrm{S}$ & $\mathrm{R} / \mathrm{S}$ \\
\hline Pseudomonas marginalis & $5 / 12$ & $4 / 12$ & $2 / 12$ & $0 / 12$ \\
\hline $\begin{array}{l}\text { Pseudomonas } \\
\text { oryzihabitans }\end{array}$ & $30 / 65$ & $20 / 65$ & $10 / 65$ & $10 / 65$ \\
\hline \multirow[t]{3}{*}{ Pseudomonas putida } & $5 / 35$ & $5 / 35$ & $5 / 35$ & $5 / 35$ \\
\hline & TIC & IMI & CIP & CHL \\
\hline & $\mathrm{R} / \mathrm{S}$ & $\mathrm{R} / \mathrm{S}$ & $\mathrm{R} / \mathrm{S}$ & $\mathrm{R} / \mathrm{S}$ \\
\hline Rahnella aquatilis & $24 / 65$ & $20 / 65$ & $12 / 65$ & $8 / 65$ \\
\hline Rahnella terrigena & $5 / 35$ & $0 / 35$ & $0 / 35$ & $0 / 35$ \\
\hline Ralstonia picketii & ND & ND & ND & ND \\
\hline Raoultella ornithinolytica & $29 / 69$ & $20 / 69$ & $10 / 69$ & $10 / 69$ \\
\hline Raoultella planticola & $15 / 35$ & $20 / 35$ & $10 / 35$ & $5 / 35$ \\
\hline Serratia fonticola & $45 / 95$ & 30/95 & $15 / 95$ & $5 / 95$ \\
\hline Serratia liquefaciens & $25 / 87$ & $32 / 87$ & $16 / 87$ & $10 / 87$ \\
\hline Serratia marcescens & $16 / 64$ & $12 / 64$ & $5 / 64$ & $2 / 64$ \\
\hline $\begin{array}{l}\text { Sphingomonas } \\
\text { parapaucimobilis }\end{array}$ & ND & ND & ND & ND \\
\hline \multirow[t]{3}{*}{ Sphingomonas melonis } & ND & ND & ND & ND \\
\hline & TIG & LIN & CIP & CHL \\
\hline & $\mathrm{R} / \mathrm{S}$ & $\mathrm{R} / \mathrm{S}$ & $\mathrm{R} / \mathrm{S}$ & $\mathrm{R} / \mathrm{S}$ \\
\hline Staphylococcus capitis & $15 / 136$ & $25 / 136$ & $20 / 136$ & $10 / 136$ \\
\hline Staphylococcus epidermidis & $60 / 168$ & $30 / 168$ & $15 / 168$ & $5 / 168$ \\
\hline $\begin{array}{c}\text { Staphylococcus } \\
\text { haemolyticus }\end{array}$ & $28 / 58$ & $15 / 58$ & $10 / 58$ & $5 / 58$ \\
\hline Staphylococcus hominis & $41 / 112$ & $23 / 112$ & $16 / 112$ & $7 / 112$ \\
\hline Staphylococcus warneri & $5 / 64$ & $15 / 64$ & $10 / 64$ & $5 / 64$ \\
\hline
\end{tabular}

CEF-cefepime; CIP-ciprofloxacin; TIC—-ticarcillin; IMI-imipenem; CHL-chloramphenicol; TEI-teicoplanin; TIG—tigecycline; LIN—linezolid; TOB—tobramycin; AMP—ampicillin; MER-meropenem. ND—not defined. Rresistant; S-sensitive.

\subsection{Antimicrobial Activity of Isolated Bee Digestive Tract Bacteriome against P. larvae}

The interactions between intestinal bacteria and pathogens of $A$. mellifera, in particular the action of intestinal bacteria against $P$. larvae, are an area of great research interest. Research on microbial composition of digestive tract of $A$. mellifera are perspective from the bee's health point of view. The research on antagonisms of $P$. larvae may promote the development of bee-friendly compounds, to protect the bees from infection with pathogens. 
All microorganisms tested showed antimicrobial activity against $P$. larvae. The strongest antimicrobial activity was shown by Lactobacillus, whereas the weakest was typical for Enterobacteriaceae (Table 5). Among the species analyzed, L. kunkei, L. crispatus, L. acidophilus were the most active against $P$. larvae. Klebsiella variicola, Ralstonia picketii, Pantotea agglomerans, Pa. vagans and Serratia liquefaciens were less active against P. larvae isolated from bee intestines. The strongest antimicrobial activity of L. kunkei, L. acidophilus and L. crispatus and the weakest antimicrobial activity of Pa. ananatis and Rahnella aquatilis were found against P. larvae CCM 4483.

Table 5. Antimicrobial activity of individual isolates against $P$. larvae in mm (mean $\pm \mathrm{SD}$ of three replicates).

\begin{tabular}{|c|c|c|}
\hline Species & P. larvae & P. larvae CСM 4483 \\
\hline Aeromonas salmonicida & $10.67 \pm 0.58$ & $10.33 \pm 0.58$ \\
\hline Arthrobacter tumbae & $9.67 \pm 1.15$ & $8.67 \pm 0.58$ \\
\hline Bacillus cereus & $14.33 \pm 0.58$ & $13.67 \pm 0.58$ \\
\hline Bacillus circulans & $14.67 \pm 1.15$ & $14.33 \pm 0.58$ \\
\hline Bacillus licheniformis & $15.67 \pm 0.58$ & $16.33 \pm 1.15$ \\
\hline Bacillus megaterium & $11.67 \pm 0.58$ & $11.33 \pm 0.58$ \\
\hline Bacillus oleronius & $10.33 \pm 1.15$ & $10.67 \pm 0.58$ \\
\hline Bacillus spp. & $9.33 \pm 0.58$ & $8.67 \pm 0.58$ \\
\hline Bacillus subtilis & $12.33 \pm 0.58$ & $11.67 \pm 0.58$ \\
\hline Bacillus thuringiensis & $12.33 \pm 1.15$ & $11.67 \pm 1.15$ \\
\hline Citrobacter spp. & $8.67 \pm 0.58$ & $6.67 \pm 1.53$ \\
\hline Citrobacter braakii & $8.33 \pm 1.53$ & $7.33 \pm 1.15$ \\
\hline Citrobacter koseri & $6.33 \pm 1.53$ & $7.67 \pm 0.58$ \\
\hline Delftia acidovorans & $11.67 \pm 1.15$ & $11.33 \pm 0.58$ \\
\hline Enterobacter aerogenes & $8.67 \pm 0.58$ & $6.67 \pm 1.53$ \\
\hline Enterobacter clocae & $8.33 \pm 1.53$ & $7.33 \pm 1.15$ \\
\hline Enterobacter kobei & $6.33 \pm 1.53$ & $7.67 \pm 0.58$ \\
\hline Enterococcus cloacae & $14.67 \pm 0.58$ & $14.33 \pm 0.58$ \\
\hline Enterococcus faecalis & $16.33 \pm 1.53$ & $16.33 \pm 0.58$ \\
\hline Escherichia coli & $15.67 \pm 0.58$ & $15.33 \pm 0.58$ \\
\hline Fructobacillus fructosus & $18.67 \pm 0.58$ & $18.33 \pm 0.58$ \\
\hline Hafnia alvei & $8.33 \pm 1.53$ & $7.33 \pm 1.15$ \\
\hline Klebsiella aerogenes & $6.33 \pm 1.53$ & $7.67 \pm 0.58$ \\
\hline Klebsiella oxytoca & $7.67 \pm 0.58$ & $8.33 \pm 0.58$ \\
\hline Klebsiella pneumoniae & $7.33 \pm 0.58$ & $6.67 \pm 0.58$ \\
\hline Klebsiella variicola & $5.33 \pm 0.58$ & $4.67 \pm 0.58$ \\
\hline Kocuria kristinae & $11.33 \pm 0.58$ & $10.67 \pm 0.58$ \\
\hline Lactobacillus acidophilus & $23.33 \pm 0.58$ & $22.67 \pm 0.58$ \\
\hline Lactobacillus agilis & $18.67 \pm 0.58$ & $18.33 \pm 0.58$ \\
\hline Lactobacillus apis & $20.33 \pm 0.58$ & $20.67 \pm 0.58$ \\
\hline Lactobacillus brevis & $19.33 \pm 0.58$ & $19.00 \pm 1.00$ \\
\hline Lactobacillus crispatus & $20.33 \pm 1.15$ & $19.67 \pm 1.15$ \\
\hline Lactobacillus jensenii & $20.33 \pm 0.58$ & $20.33 \pm 1.15$ \\
\hline Lactobacillus kunkeei & $25.67 \pm 1.15$ & $24.33 \pm 0.58$ \\
\hline Lactobacillus mellis & $18.67 \pm 1.15$ & $17.67 \pm 0.58$ \\
\hline Lactobacillus plantarum & $22.33 \pm 0.58$ & $21.67 \pm 0.58$ \\
\hline Lactobacillus spp. & $17.00 \pm 1.00$ & $17.33 \pm 0.58$ \\
\hline Lactococcus garvieae & $16.67 \pm 0.58$ & $16.33 \pm 0.58$ \\
\hline Lactococcus lactis & $17.67 \pm 0.58$ & $17.33 \pm 0.58$ \\
\hline Microbacterium pumilum & $13.67 \pm 0.58$ & $13.33 \pm 0.58$ \\
\hline Microbacterium testaceum & $12.67 \pm 0.58$ & $12.33 \pm 0.58$ \\
\hline Moraxella spp. & $8.67 \pm 0.58$ & $6.67 \pm 1.53$ \\
\hline Morganella morgani & $8.33 \pm 1.53$ & $7.33 \pm 1.15$ \\
\hline Pantotea agglomerans & $6.33 \pm 1.53$ & $7.67 \pm 0.58$ \\
\hline Pantotea ananatis & $8.67 \pm 0.58$ & $6.67 \pm 1.53$ \\
\hline Proteus mirabilis & $8.33 \pm 1.53$ & $7.33 \pm 1.15$ \\
\hline
\end{tabular}


Table 5. Cont.

\begin{tabular}{ccc}
\hline Species & P. larvae & P. larvae CCM 4483 \\
\hline Pantotea vagans & $6.33 \pm 1.53$ & $7.67 \pm 0.58$ \\
Pseudomonas marginalis & $11.33 \pm 0.58$ & $10.67 \pm 0.58$ \\
Pseudomonas oryzihabitans & $11.33 \pm 1.15$ & $11.00 \pm 1.00$ \\
Pseudomonas putida & $10.67 \pm 0.58$ & $10.33 \pm 0.58$ \\
Rahnella aquatilis & $8.67 \pm 0.58$ & $6.67 \pm 1.53$ \\
Rahnella terrigena & $8.33 \pm 1.53$ & $7.33 \pm 1.15$ \\
Ralstonia picketii & $6.33 \pm 1.53$ & $7.67 \pm 0.58$ \\
Raoultella ornithinolytica & $8.67 \pm 0.58$ & $6.67 \pm 1.53$ \\
Raoultella planticola & $8.33 \pm 1.53$ & $7.33 \pm 1.15$ \\
Serratia fonticola & $8.67 \pm 0.58$ & $6.67 \pm 1.53$ \\
Serratia liquefaciens & $8.33 \pm 1.53$ & $7.33 \pm 1.15$ \\
Serratia marcescens & $6.33 \pm 1.53$ & $7.67 \pm 0.58$ \\
Sphingomonas parapaucimobilis & $11.67 \pm 1.15$ & $11.33 \pm 0.58$ \\
Sphingomonas melonis & $10.67 \pm 0.58$ & $10.33 \pm 0.58$ \\
Staphylococcus capitis & $13.67 \pm 0.58$ & $13.33 \pm 0.58$ \\
Staphylococcus epidermidis & $14.67 \pm 0.58$ & $14.33 \pm 0.58$ \\
Staphylococcus haemolyticus & $13.67 \pm 0.58$ & $13.33 \pm 0,58$ \\
Staphylococcus hominis & $12.67 \pm 0.58$ & $12.33 \pm 0.58$ \\
Staphylococcus warneri & $11.67 \pm 0.58$ & $11.33 \pm 0.58$ \\
\hline
\end{tabular}

\subsection{Antimicrobial Activity of Essential Oils against P. larvae}

The next aim of the work was to determine the antimicrobial activity of essential oils against two strains of $P$. larvae. The highest antimicrobial activity (Table 6) was recorded for Thymus vulgaris (19.67 $\pm 1.53 \mathrm{~mm}$ and $15.67 \pm 1.53)$, Origanum vulgare ( $18.67 \pm 1.15$ and $19.00 \pm 1.00 \mathrm{~mm}$, respectively) and Pinus montana (17.67 \pm 0.58 and $17.33 \pm 0.58 \mathrm{~mm}$, respectively). The lowest antimicrobial activity was recorded for Citrus sinensis $(2.00 \pm 1.00 \mathrm{~mm})$.

Table 6. Antimicrobial activity of essential oils against $P$. larvae in $\mathrm{mm}$.

\begin{tabular}{ccc}
\hline Essential Oil & P. larvae & P. larvae CCM 4483 \\
\hline Lavandula angustifolia Mill. & $14.33 \pm 1.15$ & $15.33 \pm 0.58$ \\
Cinnamomum zeylanicum L. & $10.00 \pm 1.00$ & $12.33 \pm 2.52$ \\
Pinus montana Mill. & $17.67 \pm 0.58$ & $17.33 \pm 0.58$ \\
Mentha piperita L. & $7.33 \pm 0.58$ & $7.00 \pm 2.00$ \\
Foeniculum vulgare Mill. & $14.66 \pm 0.58$ & $14.00 \pm 0.57$ \\
Pinus sylvestris L. & $17.00 \pm 1.00$ & $17.67 \pm 0.57$ \\
Satureja hortensis L. & $12.33 \pm 0.58$ & $17.67 \pm 1.53$ \\
Origanum vulgare L. & $18.67 \pm 1.15$ & $19.00 \pm 1.00$ \\
Pimpinella anisum L. & $12.33 \pm 0.58$ & $11.67 \pm 0.58$ \\
Rosmarinus officinalis L. & $14.67 \pm 0.58$ & $10.00 \pm 1.00$ \\
Salvia officinalis L. & $14.33 \pm 0.58$ & $13.00 \pm 1.00$ \\
Abies alba Mill. & $17.33 \pm 0.58$ & $18.00 \pm 1.00$ \\
Citrus aurantium var. dulce L. & $4.33 \pm 0.58$ & $3.00 \pm 1.00$ \\
Citrus sinensis L. Osbeck. & $2.00 \pm 1.00$ & $5.33 \pm 0.58$ \\
Cymbopogon nardus L. & $8.67 \pm 0.58$ & $8.00 \pm 1.00$ \\
Mentha spicata var. crispa L. & $9.67 \pm 1.53$ & $9.33 \pm 0.57$ \\
Thymus vulgaris L. & $19.67 \pm 1.53$ & $15.67 \pm 1.53$ \\
Carvum carvi L. & $7.67 \pm 0.58$ & $5.00 \pm 0.58$ \\
Thymus serpyllum L. & $4.33 \pm 0.58$ & $7.33 \pm 0.58$ \\
Amyris balsamifera & $9.33 \pm 0.58$ & $9.67 \pm 0.58$ \\
Ocimum basilicum & $13.67 \pm 1.15$ & $14.00 \pm 1.00$ \\
Canarium luzonicum Miq. & $11.33 \pm 1.15$ & $12.33 \pm 0.58$ \\
\hline
\end{tabular}


Table 6. Cont.

\begin{tabular}{ccc}
\hline Essential Oil & P. larvae & P. larvae CCM 4483 \\
\hline Eucalyptus globulus & $16.33 \pm 1.15$ & $17.33 \pm 0.58$ \\
Gaultheria procumbens & $8.33 \pm 0.58$ & $7.33 \pm 0.58$ \\
Pelargonium graveolens & $6.67 \pm 0.58$ & $7.33 \pm 0.58$ \\
Cinnamomum caphora var. & $16.00 \pm 1.73$ & $15.67 \pm 1.15$ \\
linalolifera & $7.67 \pm 1.15$ & $7.00 \pm 1.00$ \\
Boswellia carterii & $9.67 \pm 0.58$ & $9.33 \pm 0.58$ \\
Melaleuca leucadendron & $10.33 \pm 0.58$ & $10.66 \pm 0.58$ \\
Litsea cubeba Pers. & $9.67 \pm 0.58$ & $10.00 \pm 1.00$ \\
\hline
\end{tabular}

\section{Discussion}

The highest counts of the aerobic microorganisms, Bacillus spp., Lactobacillus spp. and coliform bacteria were found in the intestine of winter bees and the lowest in the rectum of summer bees. Similar results of bacterial counts have been reported previously [10-13]. The microbiome of bees represents not only the microorganisms present in the adult worker bees, but also reflects the hive microbiota. The origin of hive microorganisms are nectar, pollen, dust and other airborne and soilborne environmental contaminants [12-14]. The excrement of honey bees and animals could be a source of microbiota during nectar harvesting. A wide variation in bacteria associated with bees have been ascribed to the external environment [15]. The bacteriota of the digestive tract of the Japanese eastern bee (Apis cerana japonica) revealed that Bacillus species could be potential antagonists for biologic control of P. larvae [16].

Non-culture studies of bee microbiome were conducted on the digestive tract or only on the middle and posterior parts of the intestines [17-25] and revealed that the pollination-based environmental microbiota and the four nectar-bearing ones are an important source of the beneficiary and potentially beneficiary microorganisms for bees [26-28]. Lactobacillus spp. were frequently found in the bee intestines and were considered the most important genus of lactic acid bacteria (LAB) in promoting animal and human health [11,29-31]. Lactobacillus spp. play significant role in feed digestibility in animals and they are important for functioning of gastrointestinal tract and accompanied immunological responses [32-37]. In our study, we did not identify species from the Bifidobacterium genus.

Antimicrobial resistance of the bacterial isolates varied in our study, depending on the genus and strain properties. Kačániová et al. [38] found resistance to tigecycline (12.5\%) and amikacin $(18.2 \%)$, gentamicin $(9.5 \%)$ and chloramphenicol $(7.2 \%)$ in their bacteriome of honey bees. Administration of antimicrobials triggers changes in the microbiome of humans and livestock, therefore, assessment of the effect of the antimicrobials on bee intestinal microorganisms is important for their health prognosis $[23,24,39,40]$ and a possible explanation of unexpected bee colony deaths [41]. The studies on microbiome diversity and its antimicrobial resistance can provide an overview on nutritional and health problems of honey bees [42].

American foulbrood (AFB) is the most destructive bacterial disease of honey bee larvae [43]. AFB is a contagious infection that begins in an individual bee larva and can cause the collapse of the entire colony because only a few spores of P. larvae are necessary to initiate the disease [44].

The use of antimicrobials, especially oxytetracycline, could protect the bees hives against infection, however, P. larvae resistance to oxytetracycline has been identified in the USA, Argentina and Canada [5,45]. Use of antimicrobials in beekeeping poses a serious risk to human health as their residues may persist in honey and other bee products [46]. Adverse effects of application of antimicrobials on the honey of honey bees [47] and on the beneficial intestinal bacteria [48] have been described.

The biologic control of AFB pathogen is considered an environmentally conscious and bee-friendly perspective. Evans and Armstrong [49,50] found that certain intestinal bacteria of A. mellifera showed antagonistic activity against $P$. larvae. Eastern Japanese bee (Apis cerana japonica), native to Japan, 
exhibited resistance against parasitic and microbial pathogens, including mite and AFB pathogen [51]. The antagonistic effect of bacteria may also depend on bacterial communities present or strains properties, including production of antimicrobial substances, e.g., bacteriocins and lysozyme and changes in $\mathrm{pH}$ as a result of organic acids production [52]. Bacteria with antagonistic properties enhance control or inhibition of pathogens. Bacillus spp. were found to exhibit bactericidal and fungicidal effects in the host gut as a result of production of various antimicrobial compounds $[53,54]$. Apis mellifera jemenitica was shown as biologically better adapted to harsh environment with higher productivity $[55,56]$.

Several natural compounds were studied for antagonistic activity against $P$. larvae in vitro [57-59], however, the identified cytotoxic effects on bees had limited their practical application. Alternatives, such as prevention and control methods of the AFB pathogen are an area of great interest. Since the ancient times, the herbal medicine and herbal extracts were applied for treatment of human and animal diseases [60]. Biologically active compounds of honey, propolis, essential oils, agents from spore of bacteria of honey and fungal extract of pollen were tested against AFB pathogen [61-65]. Of these, essential oils showed the strongest antibacterial activity against microorganisms responsible for bee diseases without toxicity on bees in vitro. The main complication in those studies is to obtain the results applicable to beekeeping related to the antimicrobial activity of essential oils and their effect on bees $[66,67]$. In our study, Thymus vulgaris was the most effective essential oil against both species of P. larvae, whereas the most effective essential oils against $P$. larvae CCM4483 were those from Pinus silvestris and Abies alba.

Tests of Melaleuca viridiflora and Cymbopogon nardus essential oils against $P$. larvae have shown an inhibition at $320 \mathrm{mg} / \mathrm{L}$ in vitro [68]. Almost all essential oils of Achyrocline satureioides, Chenopodium ambrosioide, Eucalyptus cinerea, Gnaphalium gaudichaudianum, Lippia turbinata, Marrubium vulgare, Minthostachys verticillata, Origanum vulgare, Tagetes minuta and Thymus vulgaris were effective against P. larvae strains. Eucalyptus cinerea and M. verticillata essential oils exhibited $100 \%$ efficiency in inhibiting the growth of all P. larvae strains [69]. Essential oils of Schinus molle var. areira L., Acantholippia seriphioides A. Gray, Mintosthachys mollis, Tagetes minuta L. and Lippia turbinata Griseb grown in wild in Argentina shared minimum and maximum MIC and MBC values of $200-250 \mathrm{mg} / \mathrm{L}$ and $200-300 \mathrm{mg} / \mathrm{L}$ for Andean thyme and $800-1000 \mathrm{mg} / \mathrm{L}$ and $850-1100 \mathrm{mg} / \mathrm{L}$. Andean thyme has been shown to be the most effective in vitro against $P$. larvae and could be a perspective natural alternative to the traditional antimicrobial treatment of AFB pathogen [61].

\section{Materials and Methods}

\subsection{Samples of Bees}

A total of 200 samples of Apis mellifera carnica workers were examined. Samples of bees were taken from hives from the eastern Slovakia in the Košice area $\left(48.7164^{\circ} \mathrm{N}, 21.2611^{\circ} \mathrm{E}\right)$. Bees were sampled in winter and summer, with samples from the digestive tract (intestines and rectum). examined separately. Workers of honey bees were anesthetized on ice and washed in $86 \%$ ethanol before dissection. The head or thorax of a honeybee was fixed and the entire intestine was removed by pulling the stinger using sterile dissecting forceps. The intestines and rectum were separated and collected into sterile, separate microcentrifuge tubes.

The basic dilution $\left(10^{-2}\right)$ was obtained by homogenizing $0.1 \mathrm{~g}$ of the digestive tract contents of five bees and $9.9 \mathrm{~mL}$ of peptone saline $(0.89 \%)$. Selection for groups of microorganisms followed as shown in Table 7. All agars were purchased from Oxoid (Basingstoke, United Kingdom). 
Table 7. Incubation conditions of bacteriota of the intestine of honey bees.

\begin{tabular}{|c|c|c|c|c|c|c|}
\hline \multirow{2}{*}{ Microorganisms Group } & \multirow{2}{*}{ Dilution } & \multirow{2}{*}{ Agar } & \multirow{2}{*}{$\begin{array}{l}\text { Inoculation } \\
\text { Method }\end{array}$} & \multicolumn{3}{|c|}{ Cultivation Condition } \\
\hline & & & & Relation of $\mathrm{O}_{2}$ & Temperature & Time \\
\hline TCAM & $10^{-5}-10^{-7}$ & PCA & surface & aerobic & $30^{\circ} \mathrm{C}$ & $48 \mathrm{~h}$ \\
\hline TCANM & $10^{-5}-10^{-7}$ & PCA & surface & anaerobic & $25^{\circ} \mathrm{C}$ & $48 \mathrm{~h}$ \\
\hline $\mathrm{AG}^{+}$ & $10^{-3}-10^{-6}$ & AA & surface & anaerobic & $37^{\circ} \mathrm{C}$ & $48 \mathrm{~h}$ \\
\hline Bacillus spp. & $10^{-3}-10^{-5}$ & PCA & surface & aerobic & $30^{\circ} \mathrm{C}$ & $48 \mathrm{~h}$ \\
\hline Lactobacillus spp. & $10^{-2}-10^{-6}$ & MRS & surface & aerobic & $37^{\circ} \mathrm{C}$ & $48 \mathrm{~h}$ \\
\hline Pseudomonas spp. & $10^{-3}-10^{-5}$ & Pseudomonas agar & surface & aerobic & $30^{\circ} \mathrm{C}$ & $48 \mathrm{~h}$ \\
\hline Enterococcus spp. & $10^{-3}-10^{-5}$ & Enterococcus selective agar & surface & aerobic & $37^{\circ} \mathrm{C}$ & $48 \mathrm{~h}$ \\
\hline Staphylococcus spp. & $10^{-2}-10^{-4}$ & Blood agar & surface & aerobic & $37^{\circ} \mathrm{C}$ & $48 \mathrm{~h}$ \\
\hline $\mathrm{CB}$ & $10^{-4}-10^{-6}$ & $\mathrm{McC}$ & surface & aerobic & $37^{\circ} \mathrm{C}$ & $48 \mathrm{~h}$ \\
\hline
\end{tabular}

TCAM-total counts of aerobic microorganisms; TCNANM-total counts of anaerobic microorganisms; $\mathrm{AG}^{+}$—anaerobic Gram-positive bacteria; $\mathrm{CB}$ —coliform bacteria; PCA—plate count agar; AA—anaerobic agar; MRS-Main Rogosa agar; McC-MacConkey agar.

\subsection{Identification of Bacteria}

Identification of bacteriota was performed using MALDI-TOF-MS Biotyper (Bruker Daltonics, Bremen, Germany). All the preparatory stages for the samples were carried out according to the MALDI-TOF-MS Biotyper manufacturer's recommendations. Bacterial colonies were transferred into $300 \mu \mathrm{L}$ of distilled water and $900 \mu \mathrm{L}$ of ethanol in Eppendorf tubes, which were centrifuged for $2 \mathrm{~min}$ at $14,000 \mathrm{rpm}$. The supernatant was removed, and centrifugation was repeated for the pellet, which was subsequently allowed to dry. Ten microliters of $70 \%$ formic acid and $10 \mu \mathrm{L}$ of acetonitrile were added to the dried pellet. Tubes were centrifuged for $2 \mathrm{~min}$ at $14,000 \mathrm{rpm}$ and $1 \mu \mathrm{L}$ of the supernatant was applied for identification with the MALDI-TOF. Matrix, $\alpha$-cyano-4-hydroxycinnamic acid in a volume of $1 \mu \mathrm{L}$, was added to that $1 \mu \mathrm{L}$ of supernatant and allowed to dry. The analysis was performed with a Microflex LT (Bruker Daltonics, Bremen, Germany) instrument and Flex Control 3.4 software and Biotyper Realtime Classification 3.1 with BC specific software. Confidence scores of $\geq 2.0$ and $\geq 1.7$ were the criteria for successful identification at the levels of species and genus, respectively [70].

\subsection{Antimicrobial Resistance Testing}

Antimicrobial susceptibility tests were carried out using the disc diffusion method, whereas the antimicrobial resistance of Lactobacillus spp. was assessed using MIC E-tests. Antimicrobial resistance against cefepime (CEF, $30 \mu \mathrm{g}$ ), ciprofloxacin (CIP, $10 \mu \mathrm{g}$ ), ticarcillin (TIC, $10 \mu \mathrm{g}$ ), imipenem (IMI, $10 \mu \mathrm{g}$ ), chloramphenicol (CHL, $10 \mu \mathrm{g}$ ), teicoplanin (TEI, $30 \mu \mathrm{g}$ ), tigecycline (TIG,15 $\mu \mathrm{g}$ ), linezolid (LIN, $10 \mu \mathrm{g}$ ), tobramycin (TOB, $10 \mu \mathrm{g}$ ), ampicillin (AMP, $10 \mu \mathrm{g}$ ) or meropenem (MER, $10 \mu \mathrm{g}$ ) (Oxoid, Basingstoke, UK) was examined. Bacteria strains were cultured on Muller Hinton agar for $24 \mathrm{~h}$ at $37^{\circ} \mathrm{C}$, suspended in sterile distilled water at approximately $10^{5}$ cells $/ \mathrm{mL}\left(\mathrm{A}_{620}=0.388\right.$, equivalent to a McFarland standard $)$ and used for testing. The diameters of inhibition zones were measured after incubation. Three replicates were tested for each isolate strain.

For Lactobacillus spp. strains, the MICs ( $\mu \mathrm{g} / \mathrm{mL}$ ) of AMP, MER, IMI and CHL were evaluated using the commercial E-test ${ }^{\circledR}$ (Oxoid, Basingstoke, UK). The concentrations of antimicrobials ranged from 0.016 to $256 \mu \mathrm{g} / \mathrm{mL}$. Bacterial cultures in exponential growth phase were adjusted to a suitable turbidity ( $10^{6}$ to $10^{7} \mathrm{CFU} / \mathrm{mL}$ ) and used for inoculation of iso-sensitized agar ( $90 \% \mathrm{w} / \mathrm{v}$, Oxoid, UK) supplemented with main Rogosa agar (MRS) or TPY agar (10\% w/v) (Oxoid, Basingstoke, UK). E-test strips were placed on the surface of the inoculated agar and incubated at $37^{\circ} \mathrm{C}$ for $24 \mathrm{~h}$ microaerophilically. The MIC test result was interpreted as the point at which the ellipse intersected the E-test strip as described in the E-test technical guide.

\subsection{Antimicrobial Activity of Bacterial Suspensions against P. larvae}

Bacterial strains after $24 \mathrm{~h}$ of incubation on MRS and tryptone soya agar (TSA) medium were centrifuged at $5500 \times \mathrm{g}$ for $10 \mathrm{~min}$ at $4{ }^{\circ} \mathrm{C}$ and $0.1 \mathrm{~mL}$ of the supernatant was used for detection of 
activity against $P$. larvae. A suspension $\left(0.1 \mathrm{~mL}, 10^{5} \mathrm{CFU} / \mathrm{mL}\right)$ was plated on Mueller-Hinton agar. Filter paper discs (6 mm diameter) were impregnated with $15 \mu \mathrm{L}$ of supernatant from each bacteria and placed on the P. larvae-inoculated agar. The agars were incubated initially at $4{ }^{\circ} \mathrm{C}$ for $2 \mathrm{~h}$ and then at $37^{\circ} \mathrm{C}$ for $16 \mathrm{~h}$. All tests were performed in triplicate. Filter discs impregnated with $10 \mu \mathrm{L}$ of distilled water were used as a negative control and antibiotics (amikacin, $10 \mu \mathrm{g}$ and gentamicin, $10 \mu \mathrm{g}$ ) were used as a positive control [71]. Two P. larvae isolates were tested in this study: one isolate was from bee hive and second isolate was purchased (P. larvae CCM 4483) from the Czech collection of microorganisms (Brno, Czech Republic).

\subsection{Antimicrobial Activity of Essential Oils against P. larvae}

For testing their antimicrobial activity, 30 essential oils purchased from Hanus s.r.o., Slovakia were used in the present study: Lavandula angustifolia Mill., Cinnamomum zeylanicum L., Pinus montana Mill., Mentha piperita L., Foeniculum vulgare Mill., Pinus sylvestris L., Satureja hortensis L., Origanum vulgare L., Pimpinella anisum L., Rosmarinus officinalis L., Salvia officinalis L., Abies alba Mill., Citrus aurantium var. dulce L., Citrus sinensis L. Osbeck., Cymbopogon nardus L., Mentha spicata var. crispa L., Thymus vulgaris L., Carvum carvi L., Thymus serpyllum L., Amyris balsamifera, Ocimum basilicum, Canarium luzonicum Miq., Eucalyptus globulus, Gaultheria procumbens, Pelargonium graveolens, Cinnamomum caphora var. Linalolifera, Boswellia carterii, Melaleuca leucadendron, Litsea cubeba Pers. and Melaleuca ericifolia Smith. The inoculation and testing technique was as described in Section 4.3.

\subsection{Statistical Analyses}

All measurements were made in triplicate. Statistical processing of data of the bacterial counts was performed using Microsoft Excel ${ }^{\circledR}$ software. Bacterial counts and measurements of inhibition zones were expressed as the means and standard deviation (SD). Student's $t$-test was used for calculation of significance of variability in distribution of bacteria among seasons as well as among different parts of bee gut for individual groups of analysed microorganisms. Significance of the results was considered at the following thresholds: $p \leq 0.05, p \leq 0.01, p \leq 0.001$.

\section{Conclusions}

Understanding of bacteriome inhabiting the intestine of bees has a potential to help beekeepers and promote bee health. Apis mellifera is the most important pollinator insect in means of global food security. Our studies on characterization and functional role of the bee's intestinal microbiota reveal the unique properties of $A$. mellifera bacteriota. EU prohibited antibiotics in beekeeping practice and $P$. larvae after antibiotics treatments can develop resistance. Natural antimicrobials as probiotic bacteria and essential oils can play the biggest role in control of bee pathogens.

The antimicrobials may cause an alteration in bee gut microbiota so the studies of beneficiary intestinal bacteria, which may increase colony resistance to various bee's pathogens, is a promising alternative to bee's antimicrobial treatment. Essential oils showed the inhibitory effect on $P$. larvae isolated from bees, so the application of essential oils may be expanded in beekeeping. Therefore, the present results on the antimicrobial activity of bee-beneficial bacteria and essential oils from plants can help increase the beekeepers' awareness of these possibilities and possibly reduce bee colony mortality on a global scale.

Author Contributions: Conceptualization, M.K.; data curation, M.K., M.T., J.Ž. and P.Ł.K.; investigation, M.K., M.T. and J.Ž.; methodology, M.K.; supervision, M.K.; writing—original draft, M.K., M.T., J.Ž. and P.Ł.K. All authors have read and agreed to the published version of the manuscript.

Funding: This work was supported by the grant APVV SK-BY-RD-19-0014 "The formulation of novel compositions and properties study of the polysaccharides based edible films and coatings with antimicrobial and antioxidant plant additives." 
Acknowledgments: The study was supported by the project No. 26220220180: Building Research Center "Agribiotech". Marcin Nowicki (University of Tennessee, Knoxville, USA) is gratefully acknowledged for copyediting and critical reading of this manuscript.

Conflicts of Interest: The authors declare no conflict of interest.

\section{References}

1. Jeyaprakash, A.; Hoy, M.A.; Allsopp, M.H. Bacterial diversity in worker adults of Apis mellifera capensis and Apis mellifera scutellata (Insecta: Hymenoptera) assessed using 16S rRNA sequences. J. Invertebr. Pathol. 2003, 84, 96-103. [CrossRef] [PubMed]

2. Tysset, C.; Durand, C. Contribution to the study of the intestinal microbism of healthy worker bees (Apis mellifica); reckoning and study of the constitutive groups. Bull Apic 1968, 2, 117-118.

3. Tysset, G.; Rousseau, M.; Durand, G. La présence des streptocoques du groupe D de Lancefield chez les abeilles butineuses saines (Alpis mellifica L.) L'interprétation de leur présence en bactériologie alimentaire. Bull. Acad. Vet. Fr. 1969, XLII, 173-186. [CrossRef]

4. Scardovi, V. Genus Bifidobacterium. In Bergey's Manual of Systematic Bacteriology; Williams and Wilkins: Baltimore, MD, USA, 1986.

5. Miyagi, T.; Peng, C.Y.S.; Chuang, R.Y.; Mussen, E.C.; Spivak, M.S.; Doi, R.H. Verification of Oxytetracycline-Resistant American Foulbrood Pathogen Paenibacillus larvae in the United States. J. Invertebr. Pathol. 2000, 75, 95-96. [CrossRef] [PubMed]

6. Shimanuki, H.; Knox, D.A. Susceptibility of Bacillus larvae to Terramycin. Am. bee J. 1994, 134, 125-126.

7. Michaud, V. Antibiotic residues in honey-the FEEDM view. Apiacta 2005, 40, 52-54.

8. Iwu, M.W.; Duncan, A.R.; Okunji, C.O. New antimicrobials of plant origin. In Perspectives on New Crops and New Uses; ASHS Press: Alexandria, VA, USA, 1999; pp. 457-462.

9. Dorman, H.J.D.; Deans, S.G. Antimicrobial agents from plants: Antibacterial activity of plant volatile oils. J. Appl. Microbiol. 2000, 88, 308-316. [CrossRef]

10. Rada, V.; Máchová, M.; Huk, J.; Marounek, M.; Dušková, D. Microflora in the honeybee digestive tract: Counts, characteristics and sensitivity to veterinary drugs. Apidologie 1997, 28, 357-365. [CrossRef]

11. Killer, J.; Kopečný, J.; Mrázek, J.; Rada, V.; Dubná, S.; Marounek, M. Bifidobacteria in the digestive tract of bumblebees. Anaerobe 2010, 16, 165-170. [CrossRef]

12. Kačániová, M.; Chlebo, R.; Kopernický, M.; Trakovická, A. Microflora of the honeybee gastrointestinal tract. Folia Microbiol. (Praha) 2004, 49, 169-171. [CrossRef]

13. Grubbs, K.J.; Scott, J.J.; Budsberg, K.J.; Read, H.; Balser, T.C.; Currie, C.R. Unique Honey Bee (Apis mellifera) Hive Component-Based Communities as Detected by a Hybrid of Phospholipid Fatty-Acid and Fatty-Acid Methyl Ester Analyses. PLoS ONE 2015, 10, e0121697. [CrossRef]

14. Popa, M.; Vica, M.; Axinte, R.; Glevitzky, M.; Varvara, S. Study concerning the honey qualities in Transylvania region. Ann. Univ. Apulensis Ser. Oeconomica 2009, 11, 1034-1040.

15. Nora, C.; Mahamed, A.L. Contribution to identification of the microflora of the digestive tract and pollen of Algerian honeybees: Apis mellifera intermissa and Apis mellifera sahariensis. Int. J. Curr. Microbiol. Appl. Sci. 2014, 3, 601-607.

16. Yoshiyama, M.; Kimura, K. Bacteria in the gut of Japanese honeybee, Apis cerana japonica, and their antagonistic effect against Paenibacillus larvae, the causal agent of American foulbrood. J. Invertebr. Pathol. 2009, 102, 91-96. [CrossRef] [PubMed]

17. Mohr, K.I.; Tebbe, C.C. Diversity and phylotype consistency of bacteria in the guts of three bee species (Apoidea) at an oilseed rape field. Environ. Microbiol. 2006, 8, 258-272. [CrossRef] [PubMed]

18. Babendreier, D.; Joller, D.; Romeis, J.; Bigler, F.; Widmer, F. Bacterial community structures in honeybee intestines and their response to two insecticidal proteins. FEMS Microbiol. Ecol. 2007, 59, 600-610. [CrossRef]

19. Cox-Foster, D.L.; Conlan, S.; Holmes, E.C.; Palacios, G.; Evans, J.D.; Moran, N.A.; Quan, P.-L.; Briese, T.; Hornig, M.; Geiser, D.M.; et al. A Metagenomic Survey of Microbes in Honey Bee Colony Collapse Disorder. Science (80-) 2007, 318, 283-287. [CrossRef]

20. Martinson, V.G.; Danforth, B.N.; Minckley, R.L.; Rueppell, O.; Tingek, S.; Moran, N.A. A simple and distinctive microbiota associated with honey bees and bumble bees. Mol. Ecol. 2011, 20, 619-628. [CrossRef] 
21. Disayathanoowat, T.; Young, J.P.W.; Helgason, T.; Chantawannakul, P. T-RFLP analysis of bacterial communities in the midguts of Apis mellifera and Apis cerana honey bees in Thailand. FEMS Microbiol. Ecol. 2012, 79, 273-281. [CrossRef]

22. Ahn, J.-H.; Hong, I.-P.; Bok, J.-I.; Kim, B.-Y.; Song, J.; Weon, H.-Y. Pyrosequencing analysis of the bacterial communities in the guts of honey bees Apis cerana and Apis mellifera in Korea. J. Microbiol. 2012, 50, 735-745. [CrossRef]

23. Engel, P.; Martinson, V.G.; Moran, N.A. Functional diversity within the simple gut microbiota of the honey bee. Proc. Natl. Acad. Sci. USA 2012, 109, 11002-11007. [CrossRef] [PubMed]

24. Schwarz, R.S.; Moran, N.A.; Evans, J.D. Early gut colonizers shape parasite susceptibility and microbiota composition in honey bee workers. Proc. Natl. Acad. Sci. USA 2016, 113, 9345-9350. [CrossRef] [PubMed]

25. Sabree, Z.L.; Hansen, A.K.; Moran, N.A. Independent Studies Using Deep Sequencing Resolve the Same Set of Core Bacterial Species Dominating Gut Communities of Honey Bees. PLoS ONE 2012, 7, e41250. [CrossRef]

26. Singh, S.; Saini, K.; Jain, K.L. Quantitative comparison of lipids in some pollens and their phagostimulatory effects in honey bees. J. Apic. Res. 1999, 38, 87-92. [CrossRef]

27. Singh, R.; Levitt, A.L.; Rajotte, E.G.; Holmes, E.C.; Ostiguy, N.; VanEngelsdorp, D.; Lipkin, W.I.; DePamphilis, C.W.; Toth, A.L.; Cox-Foster, D.L. RNA Viruses in Hymenopteran Pollinators: Evidence of Inter-Taxa Virus Transmission via Pollen and Potential Impact on Non-Apis Hymenopteran Species. PLoS ONE 2010, 5, e14357. [CrossRef] [PubMed]

28. McFrederick, Q.S.; Wcislo, W.T.; Taylor, D.R.; Ishak, H.D.; Dowd, S.E.; Mueller, U.G. Environment or kin: Whence do bees obtain acidophilic bacteria? Mol. Ecol. 2012, 21, 1754-1768. [CrossRef] [PubMed]

29. Olofsson, T.C.; Vásquez, A. Detection and Identification of a Novel Lactic Acid Bacterial Flora Within the Honey Stomach of the Honeybee Apis mellifera. Curr. Microbiol. 2008, 57, 356-363. [CrossRef] [PubMed]

30. Reuter, G. The Lactobacillus and Bifidobacterium microflora of the human intestine: Composition and succession. Curr. Issues Intest. Microbiol. 2001, 2, 43-53.

31. Ouwehand, A.C.; Salminen, S.; Isolauri, E. Probiotics: An overview of beneficial effects. Antonie Van Leeuwenhoek 2002, 82, 279-289. [CrossRef]

32. Sazawal, S.; Hiremath, G.; Dhingra, U.; Malik, P.; Deb, S.; Black, R.E. Efficacy of probiotics in prevention of acute diarrhoea: A meta-analysis of masked, randomised, placebo-controlled trials. Lancet Infect. Dis. 2006, 6, 374-382. [CrossRef]

33. Rafter, J.; Bennett, M.; Caderni, G.; Clune, Y.; Hughes, R.; Karlsson, P.C.; Klinder, A.; O'Riordan, M.; O'Sullivan, G.C.; Pool-Zobel, B.; et al. Dietary synbiotics reduce cancer risk factors in polypectomized and colon cancer patients. Am. J. Clin. Nutr. 2007, 85, 488-496. [CrossRef] [PubMed]

34. Younts-Dahl, S.M.; Galyean, M.L.; Loneragan, G.H.; Elam, N.A.; Brashears, M.M. Dietary Supplementation with Lactobacillus- and Propionibacterium-Based Direct-Fed Microbials and Prevalence of Escherichia coli O157 in Beef Feedlot Cattle and on Hides at Harvest. J. Food Prot. 2004, 67, 889-893. [CrossRef] [PubMed]

35. Younts-Dahl, S.M.; Osborn, G.D.; Galyean, M.L.; Rivera, J.D.; Loneragan, G.H.; Brashears, M.M. Reduction of Escherichia coli $\mathrm{O} 157$ in Finishing Beef Cattle by Various Doses of Lactobacillus acidophilus in Direct-Fed Microbials. J. Food Prot. 2005, 68, 6-10. [CrossRef] [PubMed]

36. Nocek, J.E.; Kautz, W.P. Direct-Fed Microbial Supplementation on Ruminal Digestion, Health, and Performance of Pre- and Postpartum Dairy Cattle. J. Dairy Sci. 2006, 89, 260-266. [CrossRef]

37. Chaucheyras-Durand, F.; Madic, J.; Doudin, F.; Martin, C. Biotic and Abiotic Factors Influencing In Vitro Growth of Escherichia coli O157:H7 in Ruminant Digestive Contents. Appl. Environ. Microbiol. 2006, 72, 4136-4142. [CrossRef]

38. Kačániová, M.; Gasper, J.; Brindza, J.; Schubertová, Z.; Ivanišová, E. Bacteria Of Apis Mellifera Gastrointestinal Tract: Counts, Identification And Their Antibiotic Resistance. In Agrobiodiversity for Improving Nutrition, Health and Life Quality; Agrobionet: Nitra, Slovakia, 2017; pp. 210-215.

39. Koch, H.; Schmid-Hempel, P. Socially transmitted gut microbiota protect bumble bees against an intestinal parasite. Proc. Natl. Acad. Sci. USA 2011, 108, 19288-19292. [CrossRef]

40. Li, J.; Powell, J.E.; Guo, J.; Evans, J.D.; Wu, J.; Williams, P.; Lin, Q.; Moran, N.A.; Zhang, Z. Two gut community enterotypes recur in diverse bumblebee species. Curr. Biol. 2015, 25, R652-R653. [CrossRef]

41. Jones, B.M.; Wcislo, W.T.; Robinson, G.E. Developmental Transcriptome for a Facultatively Eusocial Bee, Megalopta genalis. G3 Genes Genomes Genet. 2015, 5, 2127-2135. [CrossRef] 
42. Potts, S.G.; Biesmeijer, J.C.; Kremen, C.; Neumann, P.; Schweiger, O.; Kunin, W.E. Global pollinator declines: Trends, impacts and drivers. Trends Ecol. Evol. 2010, 25, 345-353. [CrossRef]

43. Genersch, E.; Forsgren, E.; Pentikäinen, J.; Ashiralieva, A.; Rauch, S.; Kilwinski, J.; Fries, I. Reclassification of Paenibacillus larvae subsp. pulvifaciens and Paenibacillus larvae subsp. larvae as Paenibacillus larvae without subspecies differentiation. Int. J. Syst. Evol. Microbiol. 2006, 56, 501-511. [CrossRef]

44. Genersch, E.; Ashiralieva, A.; Fries, I. Strain- and Genotype-Specific Differences in Virulence of Paenibacillus larvae subsp. larvae, a Bacterial Pathogen Causing American Foulbrood Disease in Honeybees. Appl. Environ. Microbiol. 2005, 71, 7551-7555. [CrossRef] [PubMed]

45. Evans, J.D. Diverse origins of tetracycline resistance in the honey bee bacterial pathogen Paenibacillus larvae. J. Invertebr. Pathol. 2003, 83, 46-50. [CrossRef]

46. Mutinelli, F.; Rademacher, E. The use of drugs to control varroosis in honey bee colonies and European legislation: The current situation. Bee World 2003, 84, 55-59. [CrossRef]

47. Thompson, H.M.; Waite, R.J.; Wilkins, S.; Brown, M.A.; Bigwood, T.; Shaw, M.; Ridgway, C.; Sharman, M. Effects of European foulbrood treatment regime on oxytetracycline levels in honey extracted from treated honeybee (Apis mellifera) colonies and toxicity to brood. Food Addit. Contam. 2005, 22, 573-578. [CrossRef] [PubMed]

48. Vásquez, A.; Forsgren, E.; Fries, I.; Paxton, R.J.; Flaberg, E.; Szekely, L.; Olofsson, T.C. Symbionts as Major Modulators of Insect Health: Lactic Acid Bacteria and Honeybees. PLoS ONE 2012, 7, e33188. [CrossRef]

49. D Evans, J.; Armstrong, T.-N. Inhibition of the American foulbrood bacterium, Paenibacillus larvae larvae, by bacteria isolated from honey bees. J. Apic. Res. 2005, 44, 168-171. [CrossRef]

50. Evans, J.D.; Armstrong, T.-N. Antagonistic interactions between honey bee bacterial symbionts and implications for disease. BMC Ecol. 2006, 6, 4. [CrossRef]

51. Chen, Y.-W.; Wang, C.-H.; An, J.; Kai-Kuang, H. Susceptibility of the Asian honey bee, Apis cerana, to American foulbrood, Paenibacillus larvae larvae. J. Apic. Res. 2000, 39, 169-175. [CrossRef]

52. De Vuyst, L.; Leroy, F. Bacteriocins from Lactic Acid Bacteria: Production, Purification, and Food Applications. J. Mol. Microbiol. Biotechnol. 2007, 13, 194-199. [CrossRef]

53. Alippi, A.M. Is Terramycin ${ }^{\circledR}$ losing its effectiveness against AFB? Bee Biz 2000, 11, 27-29.

54. Martirani, L.; Varcamonti, M.; Naclerio, G.; De Felice, M. Purification and partial characterization of bacillocin 490, a novel bacteriocin produced by a thermophilic strain of Bacillus licheniformis. Microb. Cell Fact. 2002, 1, 1. [CrossRef] [PubMed]

55. Alqarni, A.; Hannan, M.; Owayss, A.; Engel, M. The indigenous honey bees of Saudi Arabia (Hymenoptera, Apidae, Apis mellifera jemenitica Ruttner): Their natural history and role in beekeeping. Zookeys 2011, 134, 83-98. [CrossRef]

56. Alghamdi, A. The Comprehensive Study of the Mite, Varroa Destructor on Honeybees Apis mellifera; Indigenous and Imported; Bee Research Unit, PhD Department, College of Food and Agriculture Sciences, King Saud University: Riyadh, Saudi Arabia, 2002.

57. Ansari, M.J.; Al-Ghamdi, A.; Usmani, S.; Al-Waili, N.; Nuru, A.; Sharma, D.; Khan, K.A.; Kaur, M.; Omer, M. In vitro evaluation of the effects of some plant essential oils on Paenibacillus larvae, the causative agent of American foulbrood. Biotechnol. Biotechnol. Equip. 2016, 30, 49-55. [CrossRef]

58. Erler, S.; Moritz, R.F.A. Pharmacophagy and pharmacophory: Mechanisms of self-medication and disease prevention in the honeybee colony (Apis mellifera). Apidologie 2016, 47, 389-411. [CrossRef]

59. Kuzyšinová, K.; Mudroňová, D.; Toporčák, J.; Molnár, L.; Javorský, P. The use of probiotics, essential oils and fatty acids in the control of American foulbrood and other bee diseases. J. Apic. Res. 2016, 55, 386-395. [CrossRef]

60. Kaufman, P.; Dayanandan, P.; Li, C.; McKenzie, M.; Hoyt, J.; Kirakosyan, A. The Uses of Plant Natural Products by Humans and Risks Associated with Their Use. In Natural Products from Plants, 2nd ed.; CRC Press: Boca Raton, FL, USA, 2006; pp. 441-473.

61. Fuselli, S.R. Antimicrobial activity of some Argentinean wild plant essential oils against Paenibacillus larvae larvae, causal agent of American foulbrood (AFB). J. Apic. Res. 2006, 6, 2-7. [CrossRef]

62. Fuselli, S.R.; de la Rosa, S.B.G.; Eguaras, M.J.; Fritz, R. Susceptibility of the Honeybee Bacterial Pathogen Paenibacillus larvae to Essential Oils Distilled from Exotic and Indigenous Argentinean Plants. J. Essent. Oil Res. 2008, 20, 464-470. [CrossRef] 
63. Gende, L.B.; Floris, I.; Fritz, R.; Eguaras, M.J. Antimicrobial activity of cinnamon (Cinnamomum zeylanicum) essential oil and its main components against Paenibacillus larvae from Argentine. Bull. Insectol. 2008, 61, 1-4.

64. Antúnez, K.; Harriet, J.; Gende, L.; Maggi, M.; Eguaras, M.; Zunino, P. Efficacy of natural propolis extract in the control of American Foulbrood. Vet. Microbiol. 2008, 131, 324-331. [CrossRef]

65. Alippi, A.M.; Reynaldi, F.J. Inhibition of the growth of Paenibacillus larvae, the causal agent of American foulbrood of honeybees, by selected strains of aerobic spore-forming bacteria isolated from apiarian sources. J. Invertebr. Pathol. 2006, 91, 141-146. [CrossRef]

66. Colin, M.E.; de Lahitte, J.D.; Larribau, E.; Boué, T. Activité des huiles essentielles de Labiées sur Ascophaera apis et traitement d'un rucher. Apidologie 1989, 20, 221-228. [CrossRef]

67. Floris, I.; Carta, C. In vivo activity of Cinnamomum zeylanicum Nees essential oil against Bacillus larvae White. Apicoltura 1990, 57-61.

68. Fuselli, S.R.; García de la Rosa, B.; Eguaras, M.J.; Fritz, R. In vitro antibacterial effect of exotic plants essential oils on the honeybee pathogen Paenibacillus larvae, causal agent of American foulbrood. Spanish J. Agric. Res. 2010, 8, 651-657. [CrossRef]

69. González, M.J.; Marioli, J.M. Antibacterial activity of water extracts and essential oils of various aromatic plants against Paenibacillus larvae, the causative agent of American Foulbrood. J. Invertebr. Pathol. 2010, 104, 209-213. [CrossRef]

70. Rovná, K.; Ivanišová, E.; Žiarovská, J.; Ferus, P.; Terentjeva, M.; Kowalczewski, P.Ł.; Kačániová, M. Characterization of Rosa canina Fruits Collected in Urban Areas of Slovakia. Genome Size, iPBS Profiles and Antioxidant and Antimicrobial Activities. Molecules 2020, 25, 1888. [CrossRef] [PubMed]

71. Kačániová, M.; Gasper, J.; Terentjeva, M.; Kunová, S.; Kluz, M.; Puchalski, C. Antibacterial Activity of Bees Gut Lactobacilli against Paenibacillus Larvae In Vitro. Adv. Res. Life Sci. 2018, 2, 7-10. [CrossRef]

(C) 2020 by the authors. Licensee MDPI, Basel, Switzerland. This article is an open access article distributed under the terms and conditions of the Creative Commons Attribution (CC BY) license (http://creativecommons.org/licenses/by/4.0/). 